Apuntes Universitarios, 2020: 10(4), octubre-diciembre

ISSN: 2304-0335 DOI:https://doi.org/10.17162/au.v10i4.501

\title{
Importancia de los estilos de liderazgo: un abordaje de revisión teórica
}

\author{
Importance of leadership styles: a theoretical review approach \\ Luis Alberto Geraldo Campos, ${ }^{1}$ Alicia Roxana Mera Sánchez ${ }^{2}$ y \\ Esther Rocha Perez ${ }^{3 a}$ \\ Universidad Peruana Unión, Lima, Perú ${ }^{123}$ \\ iD ORCID ID: https://orcid.org/0000-0002-8366-689X ${ }^{1}$ \\ ORCID ID: https://orcid.org/0000-0002-0442-5335 \\ D ORCID ID: https://orcid.org/0000-0002-1312-15953
}

Recibido: 03 de enero de 2020

Aceptado: 18 de junio 2020

\begin{abstract}
Resumen
El presente estudio describe la importancia de los estilos de liderazgo desde un abordaje de revisión teórica. El análisis teórico de los estilos de liderazgo debe necesariamente partir de las teorías sobre el liderazgo en sí mismo, a fin de comprender este fenómeno en un contexto psicosocial adecuado. Esta revisión muestra que el abordaje teórico del liderazgo se ha planteado desde varios enfoques y que éstos, a su vez, han evolucionado a través del tiempo, desde principios del siglo XX hasta nuestros días. Comprender esta evolución conceptual es la clave para alcanzar una descripción precisa de los estilos de liderazgo y conocer sus aplicaciones concretas en las organizaciones. Los hallazgos, en base a la teoría consultada, indican que la importancia de los estilos de liderazgo radica en el grado de sinergia que desarrollan, al preocuparse tanto por las personas como en los objetivos de la organización liderada; además, los estilos de liderazgo más importantes son aquellos que orientan la conducta de los líderes a través de estrategias diseñadas y aplicables a una determinada cantidad de casos específicos, es decir a un tipo particular de organización.
\end{abstract}

Palabras clave: Estilos de liderazgo, liderazgo autoritario, liderazgo transaccional, liderazgo transformacional, líder.

${ }^{\mathrm{a}}$ Correspondencia al autor

E-mail: esther_13piscis@hotmail.com 


\begin{abstract}
The present study describes the importance of leadership styles from a theoretical review approach. The review of theories of leadership styles must be determined from theories of leadership itself, in order to understand this phenomenon in a suitable psychosocial context. This review shows that the theoretical approach to leadership has been approached from various perspectives and what criteria, in turn, have evolved over time, from the beginning of the 20th century to the present day. Understanding this conceptual evolution is the key to achieving an accurate description of leadership styles and knowing their specific applications in organizations. The findings, based on the theory consulted, indicate that the importance of leadership styles lies in the degree of synergy they have, as they care about both the people and the objectives of the led organization; Furthermore, the most important leadership styles are those that guide the conduct of leaders through specific strategies and determined to a limited number of specific cases, that is, a particular type of organization.
\end{abstract}

Keywords: Leadership styles; authoritarian leadership; transactional leadership; transformational leadership; Leader.

\title{
Introducción
}

El liderazgo en el ámbito organizacional es clave y crucial para el funcionamiento adecuado de la organización; por ende, alcanzar el éxito o en consecuencia llevarla al fracaso, depende de determinadas cualidades que tiene el líder (de Mello, 2015; Gemeda y Lee, 2020; Pautt, 2011; Saiyed, 2019). De acuerdo a Northouse (2007), desde inicios del siglo XX el liderazgo parte desde los enfoques teóricos de rasgos y conductuales, luego surgieron otros enfoques como el situacional y el transformacional que hasta ahora se siguen estudiando en diferentes escenarios, en ese sentido. ¿Realmente algún tipo o estilo de liderazgo es mejor que otro? ¿Será que los estilos deben darse o emplearse de acuerdo al contexto, tiempo y situación para determinar su importancia? Preguntas como estas surgen en la mente de los investigadores.

La importancia de este estudio radica en el liderazgo como tema principal de la cultura y clima organizacional, por lo que el líder es el generador principal de la calidad de estos (Carbó y Pérez, 1996; Serrano y Portalanza, 2014). Sin embargo, la existencia de diversos enfoques y estilos de liderazgo surgidos durante el tiempo, ha llevado a diversos investigadores optar por estudiar determinado enfoque. Además, resulta de particular interés indagar sobre las funciones de los líderes y los múltiples estilos de liderazgo que pueden encontrarse, teórica y concretamente, en cualquier organización humana.

Para ello, la presente revisión describe la importancia de los estilos de liderazgo, y de forma complementaria, evalúa la aplicabilidad de las mejores estrategias para las organizaciones. En ese sentido, el método usado en la presente revisión se caracteriza por utilizar un método de análisis y síntesis en base a la revisión bibliográfica de diversos autores. 
Luego, se define la noción de liderazgo a partir de las diferentes teorías sobre éste a lo largo del tiempo, y cómo los estilos de liderazgo son comprendidos por las mismas. Finalmente, se realiza una corta, pero trascendental evaluación de los estilos descritos en relación con las características principales de las organizaciones, a fin de optar por los estilos más adecuados a ser aplicados.

\section{El liderazgo}

Antes de iniciar la indagación sobre las teorías de los estilos de liderazgo, es necesario definir la noción misma de liderazgo. De acuerdo a Cąliş y Buÿükakinci (2019) "la persona que utiliza su influencia, capacidad y conocimiento para guiar a los grupos en el logro de estos objetivos se define como líder" (p. 1053); en consecuencia, un líder efectivo mejora la productividad de los colaboradores (Madanchian y Taherdoost, 2019). El liderazgo constituye un proceso altamente interactivo y que implica el establecimiento de una dirección, visión y estrategias a fin de arribar a una meta determinada, orientando a las personas con una motivación permanente (French y Bell, 1999). Por tanto, el liderazgo puede concebirse como el ejercicio de las cualidades del líder que determina una influencia en otras personas a fin de cumplir ciertos objetivos.

Debido a que gran parte de la literatura concerniente a la teorización y práctica del liderazgo proviene de Estados Unidos, su influencia en el mundo hispanohablante es, por lo menos, problemática. Esto en razón de que el término inglés leadership se ha traducido por mucho tiempo sin mayor diferenciación tanto como dirección o como liderazgo. Ambos conceptos, si bien están relacionados principalmente en el campo de la administración, poseen connotaciones diferentes y necesitan ser tratados bajo marcos conceptuales específicos (Nahavandi, 2002; Sánchez, 2008); Así pues, la dirección es un concepto más reducido, asociado a la gestión administrativa; mientras que el liderazgo debe entenderse en el marco del comportamiento organizacional en general.

Los líderes se concentran en influir en las personas que orientan, mientras que los directivos están encargados de los procesos que mantienen a la organización en adecuado funcionamiento (Bennis y Nanus, 2008). Cabe señalar que el carácter y las virtudes del líder juegan un rol importante en varios estilos de liderazgo, como el ético, el de servicio y el transformacional (Hendriks, et al., 2020); esto permite que existan muchas características comunes entre líderes y directivos, por lo que siempre es necesario analizar el contexto particular de la organización (Pautt, 2011). La tabla 1 presenta una síntesis de las principales teorías sobre el liderazgo a lo largo del tiempo. 


\section{Tabla 1}

Teorías sobre el liderazgo

\begin{tabular}{|c|c|c|}
\hline Siglo & Teorías & $\begin{array}{c}\text { Aporte o características de la } \\
\text { teoría }\end{array}$ \\
\hline $\begin{array}{l}\text { Principios de los años } 20 \\
\text { del s. XX }\end{array}$ & Enfoque personalista & $\begin{array}{l}\text { Énfasis en las características } \\
\text { personales de los líderes: } \\
\text { personalidad, capacidad de } \\
\text { influencia, etc. }\end{array}$ \\
\hline $\begin{array}{l}\text { Mediados del s. XX hasta } \\
\text { inicios del s. XXI }\end{array}$ & Enfoque situacional o funcionalista & $\begin{array}{l}\text { Énfasis en la conducta del líder, } \\
\text { más que en su personalidad. }\end{array}$ \\
\hline $\begin{array}{l}\text { Inicios del s. XXI hasta } \\
\text { nuestros días }\end{array}$ & $\begin{array}{l}\text { Teorías contingenciales: } \\
\text { - Teoría de la contingencia de Fiedler } \\
\text { - Teoría de la trayectoria-meta ("path-goal } \\
\text { theory”) de Robert House y colaboradores. } \\
\text { - Teoría situacional del Liderazgo de Paul } \\
\text { Hersey y Kenneth Blanchard. } \\
\text { - Teoría de la decisión normativa de Vroom- } \\
\text { Yetton (1973) y Vroom-Jago (1990). }\end{array}$ & $\begin{array}{l}\text { Énfasis en la interacción de un } \\
\text { tipo de líder y las características } \\
\text { de la situación en la que ejecuta } \\
\text { su tarea. }\end{array}$ \\
\hline
\end{tabular}

Fuente: Northouse (2007)

Habiendo recorrido rápidamente la evolución teórica de la noción de liderazgo, éste puede entenderse como un proceso de influencia social a través del cual el líder busca la participación voluntaria de sus subordinados, en un esfuerzo por alcanzar objetivos organizacionales (Chiavenato, 2000). Otros investigadores han hecho énfasis en que el liderazgo debe concebirse como una influencia interpersonal de carácter natural que alcanza un individuo o grupo de individuos sobre otros, a fin de llegar al éxito de una organización (Davis y Newstron, 2004; Mullins, 2004; Shermerhon, Hunt, y Osbor, 2004). Resulta claro que el comportamiento de un líder puede afectar el grado de confianza y satisfacción de los empleados o subordinados hacia la organización, y de esta relación dependerá su grado de compromiso en su desarrollo.

\section{Enfoques teóricos de liderazgo}

El estilo de liderazgo (ing. leadership style) se concibe como el patrón de comportamiento que caracteriza a un líder (Dubrin, 2015). Se trata de la performance que este manifiesta y ejecuta durante el ejercicio de su orientación. Puede comprenderse que existe una estrecha relación entre cultura organizacional y estilos de liderazgo, puesto que lo último puede afectar el compromiso organizacional de las partes involucradas y, en consecuencia, la perfomance laboral. Para que esta influencia sea positiva, el estilo de liderazgo debe estar culturalmente orientado, abarcando creencias y tradiciones de los colaboradores, sus normas y valores, así como sus preocupaciones más importantes (Tsai, Wu, y Chung, 2009). Dicho de otro modo, los 
estilos de liderazgo deben ser seleccionados y adaptados de acuerdo a la organización, situación, grupo o individuos que se maneja (Rowe, 2007). En lo concerniente a este punto-y a las teorías mencionadas en la tabla $1-$, la tabla 2 resume los enfoques, descripción y principales elementos del liderazgo que han surgido durante los últimos siglos.

\section{Tabla 2}

\section{Enfoque, descripción y elementos del liderazgo}

\begin{tabular}{|c|c|c|}
\hline Enfoque & Descripción & Elementos \\
\hline $\begin{array}{l}\text { Enfoque de los } \\
\text { rasgos }\end{array}$ & $\begin{array}{l}\text { Se centra en identificar las características personales de } \\
\text { un líder. }\end{array}$ & $\begin{array}{l}\text { Líder } \\
\text { Comunicación }\end{array}$ \\
\hline $\begin{array}{l}\text { Enfoque de } \\
\text { comportamiento }\end{array}$ & $\begin{array}{l}\text { Se centra en el comportamiento de las personas que son } \\
\text { líderes y de las que no lo son. En este enfoque surgen tres } \\
\text { estilos de liderazgo: Autocrático o Autoritario, } \\
\text { Democrático o Participativo y el estilo Laissez-Faire. }\end{array}$ & $\begin{array}{l}\text { Líder, subordinado, } \\
\text { autoridad, resultado, } \\
\text { toma de decisiones, } \\
\text { comunicación } \\
\text { motivación }\end{array}$ \\
\hline $\begin{array}{l}\text { Enfoque de la } \\
\text { contingencia }\end{array}$ & $\begin{array}{l}\text { Se plantea que en todo los momentos o situaciones se } \\
\text { requiere de un liderazgo. Es decir, depende de la situación } \\
\text { para que surja un determinado estilo de liderazgo. }\end{array}$ & $\begin{array}{l}\text { Líder, seguidores, } \\
\text { situación, contexto, } \\
\text { organización, toma de } \\
\text { decisiones, objetivos y } \\
\text { comunicación }\end{array}$ \\
\hline $\begin{array}{l}\text { Enfoque } \\
\text { emergente }\end{array}$ & $\begin{array}{l}\text { Hace referencia a dos principales tipos de liderazgo: el } \\
\text { liderazgo transformacional y transaccional. Estos estilos } \\
\text { se dan cuando los lideres transforman a las personas que } \\
\text { lo siguen y en efecto estos reciben algo por lo que se } \\
\text { genera una transacción entre sí. }\end{array}$ & $\begin{array}{l}\text { Líder, seguidores, } \\
\text { organización, } \\
\text { motivación, cliente y } \\
\text { objetivos }\end{array}$ \\
\hline
\end{tabular}

Fuente: Elaborado en base a García-Solarte (2015)

Cada enfoque descrito en la tabla 2 ha ido aportando en gran medida al liderazgo en los diversos escenarios, por lo que, a pesar de ello, se sigue estudiando este constructo. A partir de los enfoques propuestos, han venido surgiendo diversas posturas de estilos de liderazgo, algunos con mayor impacto que otros, pero los constantes cambios han permitido la adaptación e incluso la necesidad de actualizar el concepto mismo.

Es preciso señalar que se requiere de tres nociones claves en cualquier estilo de liderazgo: proceso, influencia y objetivos. Estos constituyen el núcleo básico de todos los estilos de liderazgo y permiten analizar cada uno de ellos en función de la mejor condición para una determinada organización (Northouse, 2007). Es por esto que no existe un estilo universal de liderazgo que puede calificarse como el mejor para todas las situaciones. Ciertos casos requieren diferentes estilos de liderazgo. Algunos pueden ser complejos y requieren mayor esfuerzo, otros podrían simplemente requerir un acompañamiento relativo a los agentes involucrados. En este sentido, "los estilos de liderazgo incluyen comportamientos directivos 
(directive behaviors) y comportamientos de soporte (supportive behaviors)" (Northouse, 2007, p. 94).

La anterior clasificación es de carácter general y transversal a todos los estilos de liderazgo, pero ella misma no agota las diversas clasificaciones que se han producido sobre el tema. Es por ello es importante describir y conceptualizar a una importante variedad de estilos de liderazgo en las organizaciones.

\section{Estilo de liderazgo autoritario}

De acuerdo a Sánchez (2008) "el líder autoritario se caracteriza por dar órdenes y supervisar el cabal cumplimiento de las mismas" (p.46). Se trata de un estilo dogmático, donde el líder tiene una postura vertical respecto de sus seguidores. Las recompensas y castigos se encuentran administrados únicamente por el líder, de acuerdo a su voluntad. En este sentido, y en nivel más operativo, las principales características de este estilo de liderazgo son:

\section{Tabla 3}

\section{Características del estilo de liderazgo autoritario}

\begin{tabular}{cl}
\hline Ítem & \multicolumn{1}{c}{ Característica } \\
\hline 01 & El líder determina todas las actividades. \\
02 & También determina quién se encarga de cada tarea. \\
03 & Todas las actividades son evaluadas por el líder \\
04 & Sin mayor participación de éste en las mismas \\
\hline
\end{tabular}
Fuente: Warrick (1981)

En suma, el liderazgo autoritario centraliza la dirección de la organización y no da lugar más que a su voluntad y habilidad de congregación. Este estilo se caracteriza, pues, por un alto énfasis en el desempeño laboral y un bajo énfasis en las personas o colaboradores. Generalmente, el líder autoritario asume que sus seguidores son irresponsables, perezosos, y que trabajan sin motivación, únicamente pensando en el salario recibido. Debido a estas asunciones, el líder autoritario toma el rol de un estricto planificador, directivo implacable y agente controlador cuya omnipresencia se deja notar en la presión que ejerce sobre sus seguidores. Por estas razones, este estilo de liderazgo presenta una estructura centralizada con muchos niveles de control, supervisión cerrada, trabajo estándar y comunicación de tipo $\mathrm{A} \rightarrow \mathrm{B}$. Generalmente, el líder autoritario premia la obediencia y castiga los errores, directa o indirectamente. Asimismo, los conflictos que puedan producirse al interior de la organización o grupo son suprimidos siempre a favor del líder. El trabajo, entonces, presenta un ambiente cargado de relaciones formales e impersonales (Warrick, 1981) 
Pero aquí surge una pregunta: ¿de qué manera los líderes autoritarios mantienen su posición en el largo plazo, dadas las características antes reseñadas? Generalmente, este tipo de líderes mantienen su posición limitando la participación de sus seguidores en las decisiones de la organización. Todos los líderes autoritarios desean contar con respuestas obedientes de parte de sus subordinados; ellos se mantienen hasta que sus seguidores cambien de postura y adopten la figura de competidores, con mejores ideas para la organización (Northouse, 2007). Pero todo esto tiene éxito para el líder, si éste dedica la mayoría de su tiempo a vigilar el desempeño eficiente de sus seguidores, pues su autoridad muchas veces se limita a la influencia de su presencia directa (Antonakis, Cianciolo, y Sternberg, 2004)

\section{Estilo de liderazgo democrático}

Este estilo, también denominado participativo, se halla en posición contraria al estilo autoritario. Se trata de consultar con los subordinados la planificación y desarrollo de todas las actividades, a fin de promover una participación masiva. Se trata de un líder que tiende a “involucrar a su grupo de colaboradores en el proceso de toma de decisiones, delegar la autoridad, alentar la participación en la decisión de métodos y metas de trabajo, empleando la retroalimentación como una oportunidad para guiar a sus colaboradores" (Sánchez, 2008, p. 47); es decir, se concibe la figura del líder como alguien que involucra activamente a sus subordinados en la toma de decisiones (Fierro y Villalva, 2017). Warrick (1981) ya había mencionado que un líder democrático se concentra en el desempeño de las personas bajo su mando, dando una determinada cuota de confianza y motivación a todos los agentes. Se trata de un estilo capaz de recibir abiertamente las opiniones de los seguidores y gestionarlas hacia un fin común. Ésta es la clave para entender el liderazgo democrático: la gestión de las opiniones e intereses comunes (Yoder et al., 1985). Al tener el papel de gestor, el líder divide su poder y lo comparte con su grupo de trabajadores (Bass, 2008; Robbins y Coulter, 1999). Las características principales del liderazgo democrático pueden resumirse en los siguientes puntos de la tabla 4.

Tabla 4

Características del estilo de liderazgo democrático

\begin{tabular}{cl}
\hline Ítem & \multicolumn{1}{c}{ Característica } \\
\hline 01 & Alto énfasis en los desempeños y en las personas \\
02 & $\begin{array}{l}\text { Asunción de que la mayoría de las personas son honestas y dignas de confianza, auto- } \\
\text { motivadas y responsables }\end{array}$ \\
03 & Promoción del trabajo en equipo \\
04 & Adecuada planificación y delimitación de objetivos
\end{tabular}


Estructura descentralizada de liderazgo

06 Distribución equitativa del control

07 Promoción de adecuadas condiciones de trabajo

08 Comunicación abierta de tipo $\mathrm{A} \leftrightarrow \mathrm{B}$

09 Reconocimiento del buen desempeño

10 Las acciones punitivas se toman como último recurso

11 Solución abierta de conflictos

12 Delegación de la responsabilidad

13 Utilización de evaluaciones de desempeño

Fuente: Warrick (1981)

\section{Estilo de liderazgo "laissez-faire" (dejar ser, libertad absoluta)}

También denominado liderazgo de políticas laxas, este estilo consiste en una utilización relativamente baja de la autoridad. Se trata de brindar a los seguidores una independencia que les permita ser autónomos en sus actividades. Esta autonomía les permite fijar sus propias metas y obtener sus propios medios para lograrlas. De ahí que la figura del líder involucra más que una autoridad, es un facilitador (Sánchez, 2008). En otras palabras, este tipo de liderazgo hace un énfasis equilibrado entre el desempeño laboral y las personas mismas. Además, el líder asume que sus seguidores son impredecibles, por lo que no existe interés en tratar de controlar sus diferentes características personales, ni mucho menos comprenderlas. Debido a ello, el líder deviene en alguien que mantiene un perfil bajo y no produce variaciones en su equipo de trabajo (Warrick, 1981).

Generalmente, en este tipo de liderazgo la planificación es reducida y los objetivos no están claramente definidos. El control de la organización depende en gran parte de los trabajadores. Algunos investigadores van más allá y sostienen que el liderazgo laissez-faire es en realidad un "no-liderazgo", debido a la renuncia de cualquier intervención directa por parte del líder, es decir, eluden sus responsabilidades, evitando tomar decisiones (Bass y Avolio, 1990; Chammas y Hernandez, 2019; Duran-Seguel, Gallegos, y Cabezas, 2019). Por tanto, este tipo de líderes tiende a comportarse como si abdicaran de sus responsabilidades y deberes (Lewin, Lippitt, y White, 1939), pues realizan la mínima cantidad de esfuerzo necesaria para lograr los objetivos y muestra nulo interés por sus trabajadores. En base a esto, el liderazgo laissez-faire debería ser considerado no sólo como "falta de presencia", sino también como "liderazgo cero" (Tosunoglu y Tayfur, 2016, p. 90).

Finalmente, diversos estudios han confirmado, mediante casos reales de aplicación, que el estilo de liderazgo laissez-faire provoca una falta de confianza organizacional (Fairholm, 1994; Doney, Cannon, y Mullen, 1998; Einarsen, Aasland, y Skogstad, 2007). 


\section{Estilo de liderazgo burocrático}

Consiste en la definición de procesos que se ejecutan sin variación alguna una vez que son establecidos. Se trata de una falta de flexibilidad en la dirección que, si bien ofrece seguridad a los seguidores, muchas veces no permite la apertura de los mismos a ideas y sugerencias creativas. En consecuencia, los resultados son reducidos y los subordinados se vuelven menos eficientes (Sánchez, 2008).

El primero en describir este tipo de liderazgo fue Max Weber en 1947. El estilo burocrático de liderazgo está basado en la obediencia a reglas normativas y la adherencia a determinados ejes de autoridad, es decir, más que ser un líder es un jefe (Añazco, Valdivieso, y Sánchez, 2018). Las características de este estilo son generalmente equiparadas a las del liderazgo transaccional. Las principales características de los líderes burocráticos son:

\section{Tabla 5}

\section{Características del estilo de liderazgo democrático}

\begin{tabular}{cl}
\hline Ítem & \multicolumn{1}{c}{ Característica } \\
\hline 01 & $\begin{array}{l}\text { Imposición estricta y sistemática de disciplina organizacional } \\
\text { El poder del líder reside en su posición (cargo al interior de la organización) más que en su } \\
\text { autoridad personal. }\end{array}$ \\
03 & $\begin{array}{l}\text { Los seguidores avanzan en la jerarquía de la organización a través de su habilidad para } \\
\text { adecuarse a las reglas establecidas. }\end{array}$ \\
\hline
\end{tabular}

Fuente: Foroughi citado por Sánchez (2008)

El líder burocrático, tal como el líder autoritario, determina lo que sus seguidores deben o no hacer. Generalmente, las reglas de la organización son ley para los líderes y según este aquellas deben ser tomadas como sagradas por todos los miembros. En consecuencia, se produce un ambiente de trabajo donde las personas subordinadas tienen poco o ningún espacio para la libertad de desempeño o creatividad.

\section{Estilo de liderazgo carismático}

Este consiste en el juego de la motivación. Aquí, el líder se constituye en un animador, un impulsor e inspirador de sus seguidores. Se trata, pues, de que el líder comparta sus experiencias y transmita a su equipo una serie de creencias efectivas a sí mismos, con el fin de obtener mayor eficiencia en sus trabajos respectivos (Sánchez, 2008). De acuerdo a Conger y Canungo (1996), el líder carismático hace efectivo su rol cuando su comportamiento está motivado por razones altruistas, ya sea por afinidad con la organización, poder o reconocimiento. Sin embargo, más allá de la necesidad que opera detrás de estos motivos, el 
carisma se traduce en empatía, en una preocupación por la situación de sus seguidores. Ésta es la legitimidad del liderazgo carismático (Rushton, 1982), el cual más que motivar a su equipo se exhibe como un llamado a ayudar a mejorar y tener sus objetivos claros (Añazco et al., 2018).

El líder carismático utiliza el poder que detenta sobre su equipo, pero también este último tiene poder sobre el líder. Por tanto, se trata de una relación interactiva por naturaleza. Ahora bien, la ética del líder carismático es generalmente una cuestión de cómo utilizar el poder. Esto es determinante pues si bien el liderazgo carismático puede conducir a un adecuado clima organizacional, también posee un lado peligroso. En el mejor de los casos, el carisma produce una comunidad de respeto y admiración hacia el líder. El lado peligroso del carisma reside en que el líder puede llegar a manipular a sus seguidores, muchas veces sin mayor esfuerzo (Steyrer, 1998).

Algunos investigadores consideran que los países en desarrollo necesitan de líderes carismáticos, debido a las dificultades que el desarrollo en vías de incremento implica a nivel socio-cultural. Un líder carismático, pues, es extremadamente importante en las primeras etapas de desarrollo de un país u organización, debido a que es indispensable que los objetivos comunes sean canalizados por una persona capaz de gestionarlos de la mejor manera y con el acuerdo de gran parte de la comunidad (Pfeffer, 1981; Robbins, 1992).

\section{Estilo de liderazgo informal}

Este estilo constituye el más común en cualquier organización humana. Se trata de un estilo espontáneo de liderazgo que surge gracias a la experiencia de alguien en particular, quien no posee el título oficial de líder (Sánchez, 2008). Este tipo de líder, entonces, carece de poder legítimo debido a su naturaleza. Estos líderes se basan sobre todo en relaciones de camaradería e intereses comunes y transversales a toda la comunidad.

El proceso de toma de decisiones se basa en la consulta abierta a los subordinados, por lo que los desacuerdos son siempre bien recibidos (Luria y Berson, 2012). Ahora bien, Schneier y Goktepe (1983) describen el liderazgo informal como un estilo basado en los consejos y la guía constante, más que en la represión y/o acciones punitivas. Así, los líderes informales son aquellas personas que detentan una posición de liderazgo, pero que no son reconocidos como líderes en sí mismos, ni tienen una autoridad bien definida; y si la tienen, ésta no posee rasgos de reglamentación disciplinaria sobre la organización. 


\section{Estilo de liderazgo formal.}

Las organizaciones formales deberían dar lugar a líderes formales, que detengan una autoridad reconocida al interior de una determinada organización (Luria y Berson, 2012). Este reconocimiento de la autoridad es el factor que sustenta la legitimidad del líder. De esta forma, los líderes formales tienden a presentar autoridad para la toma de decisiones, así como la habilidad de controlar el progreso laboral de sus subordinados y la gestión de acciones disciplinarias. Por ello, en este estilo de liderazgo, la comunicación es más de tipo directiva; es decir, el líder espera ser seguido y tomar decisiones de forma autocrática, más que consultiva o abierta, como era el caso en estilos anteriormente desarrollados. En consecuencia, la relación entre el líder y sus seguidos es de tipo jerárquica y para acceder a ella es necesario que los seguidores sigan todo un protocolo formal que depende directamente de su desempeño diario (Pielstick, 2000).

\section{Estilo de liderazgo orientado a las tareas}

Consiste en que el líder se concentra en realizar las tareas asignadas y busca que sus seguidores hagan lo mismo. Por tanto, se trata de dirección del trabajo por tareas, donde no hay espacio para las relaciones humanas, únicamente para el logro de objetivos. Generalmente, este estilo es muy eficiente, pues produce resultados concretos y en poco tiempo. Sin embargo, casi siempre produce incomodidad y falta de integración en el equipo (Bean-Mellinger y Seidel, 2019).

Según su fraseología en inglés: task-oriented leadership style, este estilo de liderazgo es caracterizado como de estructura básica, donde el líder se concentra de forma extensiva en el logro de ciertos objetivos y, por tanto, establece patrones de comunicación con sus seguidores adaptados a los objetivos definidos (Bass y Avolio, 1990). De acuerdo a Bean-Mellinger y Seidel (2019), un líder de este tipo se preocupa menos por poner su autoridad al servicio de las necesidades de sus seguidores, y más por encontrar soluciones técnicas y progresivas para alcanzar los objetivos previamente establecidos.

De forma operativa, un líder de este estilo pregunta siempre “¿Qué procedimientos podemos realizar para llegar a nuestros objetivos?”, en lugar de preguntar “¿Cómo podemos desarrollar la productividad de los empleados para el adecuado cumplimiento de los objetivos de la compañía?" Por tanto, un líder de este tipo es altamente lógico y operativo en sus acciones, y posee una elevada comprensión de cómo lograr sus metas concentrándose en los procedimientos necesarios para su trabajo. Además, un líder orientado a las tareas entiende que 
una tarea mayor puede involucrar varias tareas menores; en consecuencia, el líder delega el trabajo de acuerdo a un programa previamente definido (Larman, 2015)

\section{Estilo de liderazgo orientado a las personas}

Este es un estilo totalmente opuesto al liderazgo orientado a las tareas. Se trata del estilo "más humano" de todos, puesto que se concentra en las relaciones interpersonales y en la generación de buenos ambientes de trabajo (Sánchez, 2008). También denominado relationship-oriented leadership, este estilo de liderazgo hace énfasis en mostrar preocupación y respeto por sus seguidores, buscando su bienestar y soporte emocional. De acuerdo a Larman (2015), un líder con este estilo comprende la importancia de las tareas a cumplir, pero también dedica su tiempo y esfuerzo al conocimiento de las necesidades de cada uno de los integrantes de su equipo. Esto último implica la oferta de incentivos (v.g. bonus), mediación en conflictos, inversión de tiempo con los seguidores, o simplemente, una manera agradable y cordial de liderazgo.

Si se realiza un rápido análisis de las ventajas y desventajas de un liderazgo orientado a las personas, en contraposición con un liderazgo orientado a las tareas, se puede observar que cada uno depende del tipo de organización que preside. Al respecto, Friedman (2013) señala que un líder orientado a las tareas posee varias características que ayudan a asegurar que el trabajo se está haciendo de una forma eficiente.

Estos líderes generalmente crean programas claros y fáciles de seguir con requerimientos precisos y plazos establecidos. La ventaja de este estilo es que mantiene los altos estándares de calidad en la organización, con eficiencia óptima. Los seguidores que necesiten una estructura y disciplina en el trabajo, podrían muy bien situarse bajo este tipo de liderazgo. Sin embargo, la desventaja es que la estructura del líder puede conducir a una restricción de la creatividad y autonomía de los seguidores.

Un liderazgo orientado a las personas tiende a motivar a sus seguidores, porque los hace sentirse apreciados por el trabajo que hacen. Uno de los mayores beneficios de este estilo de liderazgo es que la concentración en las relaciones con los seguidores hace que éstos sientan que hacen la diferencia en su organización, es decir, son los agentes del éxito de su grupo de trabajo o empresa. Este estilo de liderazgo también presenta ciertas desventajas o desafíos fuertes (Judge, Piccolo, y Ilies, 2004; Ebert y Griffin, 2016). A veces los seguidores podrían sentir que poseen una carga demasiada pesada de responsabilidades y que necesitan más orientación o dirección. 


\section{Estilo de liderazgo situacional}

Aquí, el líder realiza una elección del estilo de liderazgo más adecuado para la situación y el equipo que debe dirigir. Constituye uno de los estilos más recomendados, debido a que es flexible. Sin embargo, muchas veces puede ocasionar una falta de equilibrio en la personalidad del líder (Sánchez, 2008). Esta teoría de liderazgo fue diseñada por Fred E. Fiedler y posteriormente por P. Hersey y K. Blanchard, cuando fusionaron sus teorías de liderazgo para crear un modelo más integral (Kwan \& Cardozo, 2018). La premisa de este modelo es que no existe un estilo de liderazgo más efectivo que los otros, sino que, por el contrario, existen diferentes estilos que son apropiados para diferentes objetivos e individuos. Los líderes exitosos, son aquellos capaces de adaptar su estilo a las personas con las que trabajan, enfatizando en las tareas y en el comportamiento de la relación con sus colaboradores (Hersey y Blanchard, 1988; Kwan y Cardozo, 2018).

\section{Estilo de liderazgo transaccional}

Se trata de un líder que establece un compromiso con su equipo, de acuerdo a las actividades a desarrollar. Generalmente, este estilo está asociado a una psicología de recompensas. A diferencia del liderazgo autoritario, en el liderazgo transaccional se asume un mismo nivel horizontal para el líder y sus seguidores (Sánchez, 2008). Los líderes con este estilo siempre están dispuestos a dar cualquier cosa a cambio de la eficiencia de sus seguidores. También denominado managerial leadership, este estilo se concentra en la supervisión, organización y desempeño laboral. Ocasionalmente pueden pasar como líderes carismáticos y pueden lograr una importancia eficiencia en la organización; los problemas surgen cuando se trata de sostener esta eficiencia, puesto que las recompensas no siempre están presentes, y si lo están, no todas las veces son bien recibidas por los seguidores (Germano, 2010)

\section{Estilo de liderazgo transformacional}

El liderazgo transformacional es el más adecuados para orientar las organizaciones hacia el cambio y la innovación (Azanza, Moriano, y Melero, 2013). En este estilo, el líder se convierte en un tipo de coach motivacional para sus seguidores. Es aquel que inspira y motiva a los seguidores a fin de que, por una parte, entreguen toda su capacidad al trabajo y a la obtención de resultados; y por otra parte, para que cada uno de los seguidores encuentre un desarrollo personal a través de sus actividades laborales (Sánchez, 2008).

Un líder transformacional es una persona que estimula e inspira a sus seguidores para permitirle lograr resultados extraordinarios a nivel laboral y personal. Estos líderes se 
preocupan por las necesidades de desarrollo de sus seguidores e intentan cambiar su estado de conciencia sobre los problemas que emergen en la organización (Odumeru y Ifeanyi, 2013). En resumen, los líderes transformacionales no sólo lideran apelando a una necesaria redundancia a sus seguidores, sino que intentan cambiarlos a nivel conductual.

Estos líderes no se dedican a utilizar el carisma o un sistema de recompensas para persuadir a sus seguidores, sino que utilizan su conocimiento de la situación y visión de largo plazo para hacer que sus seguidores se sientan profundamente involucrados en la organización. Algunos investigadores señalan que el liderazgo transformacional representa la más valiosa forma de liderazgo, puesto que los seguidores son conducidos a un cambio constante que desemboca en su transformación personal y comunitaria (Kabeyi, 2018)

\section{¿Cuál es el estilo de liderazgo más importante?}

Ahora bien, luego de haber revisado los principales estilos de liderazgo existentes en la literatura especializada, es necesario dilucidar si un estilo puede ser considerado como más importante que otros, y si éste es el caso, cuál de todos. De acuerdo a Sánchez (2008), el mejor estilo de liderazgo es aquel que realiza un alto énfasis en las tareas y un alto énfasis en las personas, lo que el autor denomina liderazgo sinérgico, ver la figura 1.

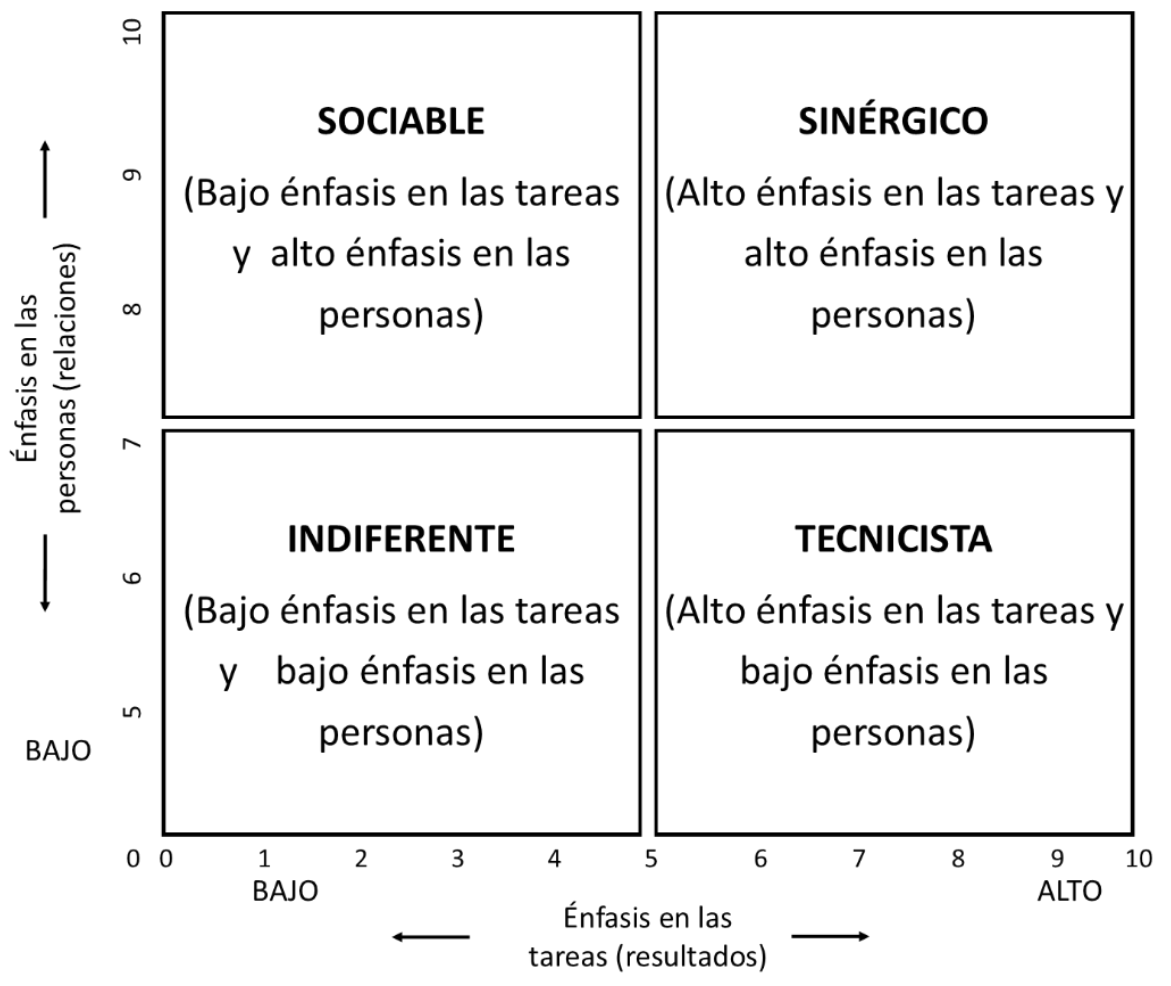

Figura 1. Modelo de estilos de liderazgo Fuente: (Sánchez, 2008, p. 112) 
Este estudio aboga por esta visión del tema, porque si bien cada organización posee un contexto determinado, es imperativo considerar ambas partes del desempeño laboral: las tareas y las personas que las desarrollan. Que este rasgo integrador lleve el nombre "sinérgico" es lo menos importante, puesto que en esta misma categoría puede entrar los estilos de liderazgo democrático y transformacional, ambos presentan un alto énfasis tanto en las personas como en las tareas a desarrollar.

Podemos señalar, entonces, que el grado de sinergia entre estas partes determina un liderazgo exitoso, en términos de desarrollo técnico y humano. A fin de cuentas, en el largo plazo, esta sinergia conducida por un líder democrático o transformacional producirá a la organización altos índices de productividad, puesto que el sector humano se sentirá como una pieza clave para su empresa y siempre estará motivado por nuevos desafíos. Indudablemente, esto es algo que otros estilos no pueden producir. Sin embargo, es posible también tener éxito en una organización sin preocuparse por las personas o por las tareas. Evidentemente, si no existe preocupación por ninguna, la organización seguirá el curso de la extinción. Por este motivo no es posible señalar en términos absolutos cuál estilo de liderazgo es más importante, dependerá del tipo de organización a la que el líder se enfrenta.

Recientemente, algunos investigadores proponen el liderazgo espiritual como un estilo capaz de abarcar múltiples enfoques e incluir a otros estilos (Reave, 2005; Smith, Minor, y Brashen, 2018). Este estilo se caracteriza por: (a) el respeto a otros valores, (b) el tratamiento justo de los seguidores, (c) la expresión de cuidado y preocupación, (d) la escucha activa y responsable de los otros, (e) la muestra de apreciación por los otros, y (f) la práctica reflexiva de las emociones (Smith et al., 2018, p. 81). Cabe añadir que esta clase de liderazgo no representa un estilo propiamente dicho de ejercer el liderazgo, sino que constituye un conjunto de rasgos particulares que pueden o no ser aplicados por los estilos que ya hemos desarrollado en el apartado anterior.

\section{Conclusiones}

Nuestro objetivo principal en la presente revisión teórica ha sido describir los principales estilos de liderazgo y establecer su grado de importancia para las organizaciones humanas de forma general. Luego de la revisión y análisis sobre los rasgos teóricos de los principales estilos de liderazgo relacionados al desarrollo organizacional y personal, puede concluirse que la importancia de los mismos radica en el grado de sinergia que desarrollan, al preocuparse tanto por las personas como en los objetivos de la organización liderada. De esta sinergia dependerá si un estilo de liderazgo es más importante que otro para una determinada organización y en un 
contexto psicosocial y socio-económico específico. Así pues, teóricamente no puede señalarse que un estilo de liderazgo es más importante que otro, ya que existe una variedad considerable de organizaciones humanas que impiden un control adecuado de todas las variables sociales involucradas. Por tanto, se concluye que los estilos de liderazgo más importantes son aquellos que orientan la conducta de los líderes a través de estrategias diseñadas para esto y aplicables a una determinada cantidad de casos específicos, es decir a un tipo particular de organización.

Por tanto, esta revisión teórica invita la apertura de nuevas investigaciones, donde las organizaciones generalmente linden con aspectos informales de organización del capital humano, surgiendo interrogantes como ¿cuál es el mejor estilo de liderazgo a ser ejecutado? Sin embargo, aplicar las mejores estrategias de liderazgo resulta de trascendental importancia para el desarrollo socio-económico de un caso en concreto, a fin de gestionar de la mejor manera los recursos, pero debe tomarse en cuenta que los estilos de liderazgo revisados anteriormente han sido inicialmente propuestos para otras realidades, como América del Norte y Europa. Por tanto, es necesario que las investigaciones futuras tomen en cuenta esta revisión y apliquen los conocimientos resultantes para el desarrollo de propuestas de liderazgo adaptadas a la realidad nacional. Únicamente de esta forma, los conocimientos aquí vertidos tendrán una aplicación transformacional, a fin de evocar uno de los rasgos fundamentales de uno de los estilos de liderazgo más contemporáneos.

\section{Referencias}

Añazco, K. A., Valdivieso, R. P., y Sánchez, Ó. W. (2018). Los estilos de liderazgo y su efecto en la satisfacción laboral. INNOVA Research Journal, 3(10), 142-148.

Antonakis, J., Cianciolo, A. T., y Sternberg, R. J. (2004). The Nature of Leadership. California, Estados Unidos: Sage Publications.

Azanza, G., Moriano, J. A., y Melero, F. (2013). Journal of Work and Organizational Psychology. Journal of Work and Organizational Psychology, 29(2), 45-50. https://doi.org/10.5093/tr2013a7.

Bass, B. M. (2008). The Bass handbook of leadership. Theory, research \& managerial applications (4th ed.). New York, Estados Unidos: Free Press.

Bass, B. M., y Avolio, B. J. (1990). Transformational leadership development: Manual for the multifactor leadership questionnaire. California, Estados Unidos: Consulting Psychologists Press.

Bean-Mellinger, B., y Seidel, M. (2019). Differences Between Task-Oriented Leaders \& Relational-Oriented Leaders. Chron. Retrieved from https://smallbusiness.chron.com/differences-between-taskoriented-leadersrelationaloriented-leaders-35998.html

Bennis, W., y Nanus, B. (2008). Líderes. Estrategias para un liderazgo eficaz. Barcelona, España: Paidós. 
Çalış, Ç., y Büyükakıncı, B. Y. (2019). Leadership Approach in Occupational Safety: Taiwan Sample. Procedia Computer Science, 158, 1052-1057. https://doi.org/10.1016/j.procs.2019.09.146

Carbó, J., \& Pérez, M. (1996). El liderazgo en el capital humano y la empresa: Los recursos humanos, la clave de la competitividad. Madrid, España: Coopers \& Lybrand.

Chammas, C. B., y Hernandez, J. M. da C. (2019). Comparing transformational and instrumental leadership. Innovation \& Management Review, 16(2), 143-160. https://doi.org/10.1108/INMR-08-2018-0064

Chiavenato, I. (2000). Administración de Recursos Humanos. Bogota, Colombia: Mc Graw Hill Interamericana.

Davis, K., y Newstron, J. (2004). Comportamiento humano en las organizaciones. México D. F., México: Editorial Mc Graw Hil.

De Mello, M. (2015). La importancia del liderazgo sostenible como una estrategia de las organizaciones. Revista Ciencias Estratégicas, 23(34), 209-218. https://doi.org/10.18566/rces.v23n34.a4

Doney, P. M., Cannon, J. P., y Mullen, M. R. (1998). Understanding the Influence of National Culture on the Development of Trust. The Academy of Management Review, 23(3), 601620. https://doi.org/10.2307/259297

Dubrin, A. J. (2015). Leadership: Research Findings, Practice, and Skills (8th ed.). Massachussetts, Estados Unidos de America: Cengage Learning.

Duran-Seguel, I. M., Gallegos, M. E., \& Cabezas, D. E. (2019). Estilos de liderazgo y su influencia en el clima laboral: caso de estudio de una empresa exportadora de alimentos. Revista ESPACIOS, 40(40), 3. Retrieved from http://www.revistaespacios.com/a19v40n40/a19v40n40p03.pdf

Ebert, R. J., \& Griffin, R. W. (2016). Business Essentials (11th ed.). Retrieved from https://www.amazon.es/kindle/dp/B01D1YBKG4/ref=rdr_kindle_ext_eos_detail

Einarsen, S., Aasland, M. S., \& Skogstad, A. (2007). Destructive leadership behaviour: A definition and conceptual model. Leadership Quarterly, 18(3), 207-216. https://doi.org/10.1016/j.leaqua.2007.03.002

Fairholm, G. W. (1994). Leadership and the Culture of Trust. Londres, Inglaterra: Greenwood Publishing Group.

Fierro, I., \& Villalva, M. (2017). El liderazgo Democrático: Una Aproximación Conceptual. INNOVA Research Journal, 2(4), 155-162. https://doi.org/10.33890/innova.v2.n4.2017.210

French, W. L., \& Bell, C. H. (1999). Organization Development: Behavioral Science Interventions for Organization Improvement (6th ed.). New Jersey, Estados Unidos: Pearson.

Friedman, M. J. (2013). Finalizing PTSD in DSM-5 : Getting Here From There and Where to Go Next. Journal of Traumatic Stress, 26(5), 548-556. https://doi.org/10.1002/jts.21840

García-Solarte, M. (2015). Formulación de un modelo de liderazgo desde las teorías $\begin{array}{lll}\text { organizacionales. } & \text { Entramado, } & \text { 60-79. }\end{array}$ https://doi.org/10.18041/entramado.2015v11n1.21111

Gemeda, H. K., y Lee, J. (2020). Leadership styles, work engagement and outcomes among 
information and communications technology professionals: A cross-national study. Heliyon, 6(4), e03699. https://doi.org/10.1016/j.heliyon.2020.e03699

Germano, M. A. (2010). Leadership Style and Organizational Impact. Library Worklife: HR ENews: ALA-Allied Professional Association, (junio). Retrieved from http://alaapa.org/newsletter/2010/06/08/hr-practice/

Hendriks, M., Burger, M., Rijsenbilt, A., Pleeging, E., y Commandeur, H. (2020). Virtuous leadership: a source of employee well-being and trust. Management Research Review, ahead-of-p(ahead-of-print). https://doi.org/10.1108/MRR-07-2019-0326

Hersey, P., y Blanchard, K. (1988). Management of Organization behavior: Utilizing Human Resources (5th ed.). New Jersey, Estados Unidos: Prentice-Hall.

Judge, T. A., Piccolo, R. F., y Ilies, R. (2004). The Forgotten Ones? The Validity of Consideration and Initiating Structure in Leadership Research. Journal of Applied Psychology, 89(1), 36-51. Retrieved from https://doi.org/10.1037/0021-9010.89.1.36

Kabeyi, M. J. B. (2018). Transformational vs transactional leadership with examples. The International Journal of Business \& Management, 6(5), 191-193. https://doi.org/10.4172/2169-

Kwan, C. K., y Cardozo, S. (2018). Estilo de liderazgo situacional predominante en las Micro y Pequeñas Empresas de Asunción, Paraguay. Academo Revista de Investigación En Ciencias Sociales y Humanidades, 5(2), 117-126. https://doi.org/10.30545/academo.2018.jul-dic.4

Larman, A. (2015, December). Task-Oriented Vs People-Oriented Leadership Styles. Ezine Articles. Retrieved from https://ezinearticles.com/?Task-Oriented-Vs-People-OrientedLeadership-Styles\&id=9253531

Lewin, K., Lippitt, R., y White, R. K. (1939). Patterns of aggressive behavior in experimentally created "social climates." The Journal of Social Psychology, 10(2), 269-299.

Luria, G., y Berson, Y. (2012). How do leadership motives affect informal and formal leadership emergence? Journal of Organizational Behavior, 34, 995-1015. https://doi.org/10.1002/job.1836

Madanchian, M., y Taherdoost, H. (2019). Assessment of Leadership Effectiveness Dimensions in Small and Medium Enterprises (SMEs). Procedia Manufacturing, 32, 1035-1042. https://doi.org/10.1016/j.promfg.2019.02.318

Mullins, L. (2004). Management and Organisational Behaviour (9th ed.). Retrieved from file:///C:/Users/admin/Downloads/epdf.pub_management-and-organisational-behaviour9th-editio.pdf

Nahavandi, A. (2002). The art and science of leadership (3rd ed.). New Jersey, Estados Unidos: Prentice Hall.

Northouse, P. . (2007). Leadership: Theory and practice (9th ed.). London: Sage publications.

Odumeru, J. A., y Ifeanyi, G. O. (2013). Transformational vs. transactional leadership theories: Evidence in literature. International Review of Management and Business Research, 2(2), 355-36. Retrieved from http://irmbrjournal.com/papers/1371451049.pdf

Pautt, G. (2011). Liderazgo y dirección: Dos conceptos distintos. Revista Facultad de Ciencias Económicas: Investigación y Reflexión, XIX(1), 213-228. Retrieved from http://www.redalyc.org/articulo.oa?id=90922732013 
Pfeffer, J. (1981). Power in Organizations (Financial Times Prentice Hall, ed.). New Jersey: Pitman.

Pielstick, C. D. (2000). Formal vs Informal Leading: A Comparative Analysis. Journal of Leadership \& Organizational Studies, 7(3), 1-14. Retrieved from http://openknowledge.nau.edu/1643/7/Pielstick_CD_2000_FrankeWPS_0004\%281\%29.pdf

Reave, L. (2005). Spiritual values and practices related to leadership effectiveness. The Leadership Quarterly, 16(5), 655-687. Retrieved from https://doi.org/10.1016/j.leaqua.2005.07.003

Robbins, S., y Coulter, M. (1999). Administración (5th ed.). México D. F., México: Prentice Hall.

Robbins, S. P. (1992). Essentials in Organizational Behaviour. New York, Estados Unidos: Prentice-Hall.

Rowe, W. G. (2007). Cases in Leadership. California, Estados Unidos: Sage Publications.

Rushton, J. P. (1982). Altruismo y sociedad: una perspectiva de aprendizaje social. Ética, 92(3), 425-446. https://doi.org/10.2307/2380730

Saiyed, A. A. M. (2019). The role of leadership in business model innovation: a case of an entrepreneurial firm from India. New England Journal of Entrepreneurship, 22(2), 70-88. https://doi.org/10.1108/neje-08-2019-0040

Sánchez, I. D. (2008). Los estilos de dirección y liderazgo: Propuesta de un modelo de caracterización y análisis. Pensamiento y Gestión, 25, 1-39. Retrieved from http://rcientificas.uninorte.edu.co/index.php/pensamiento/article/view/3194/4931

Schneier, C. E., y Goktepe, J. R. (1983). Issues in emergent leadership: The contingency model of leadership, leader sex, leader behavior (1st ed.; H. H. Blumberg, A. P. Hare, V. Kent, \& M. F. Davies, eds.). Chicester, Inglaterra: John Wiley.

Serrano, B. J., y Portalanza, A. (2014). Influencia del liderazgo sobre el clima organizacional. Suma de Negocios, 5(11), 117-125.

Shermerhon, J., Hunt, J., y Osbor, R. (2004). Comportamiento organizacional. Nueva York: Editorial Limusa Wiley.

Smith, G., Minor, M., y Brashen, H. (2018). Spiritual Leadership: A Guide to a Leadership Style That Embraces Multiple Prespectives. Journal of Instructional Research, 7, 80-89. Retrieved from https://files.eric.ed.gov/fulltext/EJ1188325.pdf

Steyrer, J. (1998). Charisma and the Archetypes of Leadership. Organization Studies, 19(5), 807-828. https://doi.org/10.1177/017084069801900505

Tosunoglu, H., y Tayfur, O. (2016). Laissez-faire leaders and organizations: How does laissezfaire leader erode the trust in organizations? Journal of Economics, Finance and Accounting, 3(1), 89-99. Retrieved from https://dergipark.org.tr/tr/download/articlefile/757422

Tsai, Y., Wu, S. W., y Chung, H. J. (2009). The Exploration of Relationship between Organizational Culture and Style of Leadership. Proceedings of the 2009 6th International Conference on Service Systems and Service Management, ICSSSM '09, 585-590. https://doi.org/10.1109/ICSSSM.2009.5174951

Warrick, D. D. (1981). Leadership styles and their consequences. Journal of Experiential 
Learning and Simulation, 3, 155-172. Retrieved from https://eric.ed.gov/?id=EJ265922

Yoder, J. D., Adams, J., Grove, S., y Priest, R. F. (1985). To Teach is to Learn: Overcoming Tokenism with Mentors. Psychology of Women Quarterly, 9(1), 119-131. https://doi.org/10.1111/j.1471-6402.1985.tb00865.x 